\title{
Black Hole Solutions in Rastall Theory
}

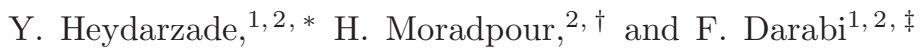 \\ ${ }^{1}$ Department of Physics, Azarbaijan Shahid Madani University, Tabriz, 53714-161, Iran \\ ${ }^{2}$ Research Institute for Astronomy and Astrophysics of Maragha (RIAAM), Maragha 55134-441, Iran
}

The Reissner-Nordström black hole solution in a generic cosmological constant background in the the context of Rastall gravity is obtained. It is shown that the cosmological constant arises naturally from the consistency of the non-vacuum field equations of the Rastall theory for a spherical symmetric spacetime, rather than its ad-hoc introduction in the usual Einstein and Einstein-Maxwell field equations. The usual Reissner-Nordström, Schwarzschild and Schwarzschild(anti)de Sitter black hole solutions in the framework of this theory are also addressed as the special independent subclasses of the obtained general solution.

Keywords: Reissner-Nordström black hole, Rastall theory of gravity.

PACS numbers: 04.50.-h, 04.20.Jb, 04.70.-s

\section{INTRODUCTION}

In the curvature-matter coupling theory of gravity [1 5], the geometry and matter fields are coupled to each other in a non-minimal way meaning that the Lagrangian of the system is not a simple summation of the Lagrangian of geometry and the matter fields. Due to this non-minimal coupling, the geometry and matter fields are affected by their mutual changes, so the ordinary energy-momentum conservation law may be invalid in this context. In fact, in this theory, the divergence of the energy-momentum tensor is related to the changes of the Ricci scalar [1 5 ].

The idea of coupling of the matter and geometry fields in a non-minimal way comes back to P. Rastall [6], who challenged the energy-momentum conservation law in the curved spacetime for the first time, a hypothesis supported by the particle creation during the cosmos evolution [7-11]. In this way, it was shown by Smalley that a prototype of Rastall's theory of gravity, in which the divergence of the energymomentum tensor is proportional to the gradient of the Ricci scalar, can be derivable from a variational principle 12. It was discussed that both the proportionality factor and the unrenormalized gravitational constant are covariantly constant, but not necessarily constant. In this regard, this theory appears as a gravitational theory with variable gravitational constant. In a cosmological context, this theory is in agreement with some observational data and leads to some interesting results [13, 14]. As an instance, the evolution of small dark matter fluctuations is identical with that of the $\Lambda C D M$ model, but in this theory dark energy is able to be clustered. This feature result in an evolution of dark matter

\footnotetext{
*Electronic address: heydarzade@azaruniv.edu; Corresponding authorpbtain the charged black hole in the cosmologi-

†Electronic address: h.moradpour@riaam.ac.ir

‡Electronic address: f.darabi@azaruniv.edu cai constant background. Finally, in section IV, we represent our concluding remarks.
}

inhomogeneities in a nonlinear regime which is different from the one in the standard $C D M$ model [15]. Thermodynamical aspects of this model in a flat FLRW universe are studied in [16, 17]. This theory is also studied in the context of the Gödeltype universe [18].

Moreover, some static spherically symmetric solutions of the Rastall field equations have been obtained. For example, by special setting of the parameters of the Rastall theory, a vacuum solution possessing the same structure as the Schwarzschildde Sitter solution in the general relativity theory obtained with a cosmological constant playing the role of source [19]. In another work, the problem of finding static spherically symmetric spaces in Rastall's theory in the presence of a free or self interacting scalar field is studied [20]. It is found that some exact solutions can be obtained in which some of these solutions are the same as the solutions which are obtained in the context of k-essence theory. Also, the asymptotically flat traversable wormhole solutions are investigated in 21] where it is shown that the parameters of Rastall theory affect the parameters of the wormhole spacetime.

In this work, our aim is to find general static spherical symmetric black hole solutions in the context of the Rastall theory of gravity as a special class of the extended theories of gravity [22]. The paper is organized as follows. In the section II, we have a review on the Rastall theory of gravity and its general remarks. In section III, we investigate the spherical symmetric black hole solutions in the Rastall theory equipped by the electromagnetic sector. Then, 


\section{RASTALL THEORY OF GRAVITY}

Based on the Rastall's hypothesis [6], for a spacetime with Ricci scalar $R$ filled by a source of $T^{\mu \nu}$ we have

$$
T_{; \mu}^{\mu \nu}=\lambda R^{, \nu},
$$

where $\lambda$ is the Rastall parameter. The Rastall field equation can be written as

$$
G_{\mu \nu}+\gamma g_{\mu \nu} R=\kappa T_{\mu \nu},
$$

where $\gamma=\kappa \lambda$ and $\kappa$ is the Rastall gravitational coupling constant. Equivalently, one can rewrite this equation in the following form

$$
G_{\mu \nu}=\kappa S_{\mu \nu},
$$

where

$$
S_{\mu \nu}=T_{\mu \nu}-\frac{\gamma T}{4 \gamma-1} g_{\mu \nu},
$$

is the effective energy-momentum tensor in which

$$
\begin{aligned}
& S^{0}{ }_{0} \equiv-\rho^{e}=-\frac{(3 \gamma-1) \rho+\gamma\left(p_{r}+2 p_{t}\right)}{4 \gamma-1} \\
& S^{1}{ }_{1} \equiv p_{r}^{e}=\frac{(3 \gamma-1) p_{r}+\gamma\left(\rho-2 p_{t}\right)}{4 \gamma-1} \\
& S^{2}{ }_{2}=S^{3}{ }_{3} \equiv p_{t}^{e}=\frac{(2 \gamma-1) p_{t}+\gamma\left(\rho-p_{r}\right)}{4 \gamma-1} .
\end{aligned}
$$

Here, $\rho^{e}, p_{r}^{e}$ and $p_{t}^{e}$ are the effective density and pressures corresponding to the source with the energymomentum tensor $T^{\mu}{ }_{\nu}$, respectively [21]. The usual Einstein general relativity can be recovered in the appropriate limit of $\lambda \rightarrow 0$. Also, it is apparent that for a traceless energy-momentum source, such as the electromagnetic source, we have $S_{\mu \nu}=T_{\mu \nu}$ which leads to $G_{\mu \nu}=\kappa T_{\mu \nu}$. This means that the Einstein solutions for $T=0$, or equivalently $R=0$, are also valid in the Rastall theory of $\kappa[13,20]$. Moreover, it should be mentioned that both of the parameter cases $\gamma=\frac{1}{4}$ and $\gamma=\frac{1}{6}$ are not allowed as discussed in [17]. Using the Newtonian limit, it is shown that the Rastall parameter $\lambda$ and Rastall gravitational coupling constant $\kappa$ diverge for $\gamma=\frac{1}{4}$ and $\gamma=\frac{1}{6}$, respectively, which does not make a physical sense 17.

In the following, we will work in the units where $\kappa=1$, leading to $\gamma=\lambda$, and therefore we have the following Rastall field equation

$$
G_{\mu \nu}=S_{\mu \nu}
$$

where

$$
S_{\mu \nu}=T_{\mu \nu}-\frac{\lambda T}{4 \lambda-1} g_{\mu \nu} .
$$

\section{BLACK HOLE SOLUTIONS IN RASTALL-MAXWELL THEORY}

In this section, we construct Rastall-Maxwell theory in order to find charged black hole solutions. We consider the following static spherical symmetric metric in the context of Rastall theory possessing the modified field equation (6)

$$
d s^{2}=-e^{\mu(r)} d t^{2}+e^{\nu(r)} d r^{2}+r^{2} d \Omega_{2}^{2},
$$

with the total energy-momentum tensor given by

$$
T_{\mu \nu}=T_{\mu \nu}^{*}+E_{\mu \nu},
$$

where

$$
T_{\mu \nu}^{*}=(\rho+p) u_{\mu} u_{\nu}+p g_{\mu \nu},
$$

is the non-vanishing trace part of the total energy momentum tensor possessing the barotropic equation of state $p=\omega \rho$, and $E_{\mu \nu}$ is the trace-free Maxwell tensor given by

$$
E_{\mu \nu}=2\left(F_{\mu \alpha} F_{\nu}^{\alpha}-\frac{1}{4} g_{\mu \nu} F^{\alpha \beta} F_{\alpha \beta}\right),
$$

where $F_{\mu \nu}$ is the antisymmetric Faraday tensor satisfying the vacuum Maxwell equations

$$
\begin{aligned}
& F_{; \mu}^{\mu \nu}=0, \\
& \partial_{[\sigma} F_{\mu \nu]}=0 .
\end{aligned}
$$

Using the equations (7) and (9), we obtain the effective energy momentum tensor $S_{\mu \nu}$ as

$$
S_{\mu \nu}=T_{\mu \nu}^{*}-\frac{\lambda T^{*}}{4 \lambda-1} g_{\mu \nu}+E_{\mu \nu} .
$$

On the other hand, spherical symmetry implies that the only non-zero components of $F^{\mu \nu}$ are $F^{01}=$ $-F^{10}$. Then, from the equations in (12), one obtains

$$
F^{01}=\frac{Q}{r^{2}} .
$$

Using the equations (8), (11) and (14), the only nonvanishing components of $E^{\mu}{ }_{\nu}$ will be

$$
E_{\nu}^{\mu}=\frac{Q^{2}}{r^{4}} e^{\mu+\nu} \operatorname{diag}(-1,-1,1,1) .
$$

Then, the effective energy momentum tensor components take the following form

$$
\begin{aligned}
& S_{0}^{0}=-\frac{(3 \lambda-1) \rho+3 \lambda p}{4 \lambda-1}-\frac{Q^{2}}{r^{4}} e^{\mu+\nu}, \\
& S^{1}{ }_{1}=\frac{(\lambda-1) p+\lambda \rho}{4 \lambda-1}-\frac{Q^{2}}{r^{4}} e^{\mu+\nu}, \\
& S^{2}{ }_{2}=S^{3}{ }_{3}=\frac{(\lambda-1) p+\lambda \rho}{4 \lambda-1}+\frac{Q^{2}}{r^{4}} e^{\mu+\nu} .
\end{aligned}
$$


Then, the 00 component of the Rastall field equation (3) yields

$$
\frac{e^{-\nu}}{r^{2}}\left(1-r \nu^{\prime}-e^{\nu}\right)=-\frac{(3 \lambda-1) \rho+3 \lambda p}{4 \lambda-1}-\frac{Q^{2}}{r^{4}} e^{\mu+\nu},
$$

and 11 component gives

$$
\frac{e^{-\nu}}{r^{2}}\left(1+\mu^{\prime} r-e^{\nu}\right)=\frac{(\lambda-1) p+\lambda \rho}{4 \lambda-1}-\frac{Q^{2}}{r^{4}} e^{\mu+\nu} .
$$

Finally, the 22 and 33 components lead to

$$
\begin{array}{r}
\frac{e^{-\nu}}{4 r}\left(2 \mu^{\prime}-2 \nu^{\prime}+r \mu^{\prime} \nu^{\prime}+2 \mu^{\prime \prime} r+\mu^{\prime 2} r\right) \\
=\frac{(\lambda-1) p+\lambda \rho}{4 \lambda-1}+\frac{Q^{2}}{r^{4}} e^{\mu+\nu} .
\end{array}
$$

One can consider the usual special class of the spherical symmetric metrics as $\nu(r)=-\mu(r)$. Consequently, by setting $\nu(r)=-\ln (1-f(r))$, the 00 component of the Rastall field equation (17) yields

$$
-f(r)-r f^{\prime}(r)=-\frac{(3 \lambda-1) \rho+3 \lambda p}{4 \lambda-1} r^{2}-\frac{Q^{2}}{r^{2}},
$$

and 11 component gives

$$
-f(r)-r f^{\prime}(r)=\frac{(\lambda-1) p+\lambda \rho}{4 \lambda-1} r^{2}-\frac{Q^{2}}{r^{2}} .
$$

Finally, the 22 and 33 components lead to

$$
-r f^{\prime}(r)-\frac{1}{2} r^{2} f^{\prime \prime}=\frac{(\lambda-1) p+\lambda \rho}{4 \lambda-1} r^{2}+\frac{Q^{2}}{r^{2}} .
$$

It is clear that the left hand sides of the equations (20) and (21) are the same. Then, the consistency for the right hand sides of these equations requires that $4 \lambda=1$ or the matter field described by (10) with $\rho \neq 0$ takes the equation of state parameter $\omega=-1$. For the case of $\rho=p=0$, these equations are trivially consistent. The first possibility, i.e $4 \lambda=1$, is ruled out based on the discussions in [21]. Regarding the second possibility, the black hole surrounding matter has an effective behavior as the same as the cosmological constant. It is easy to verify that the equations (20) and (22) are satisfied by the metric function $f(r)$ given by

$$
f(r)=\frac{2 M}{r}-\frac{Q^{2}}{r^{2}}+h(r),
$$

where $M$ and $Q$ are the black hole mass and charge respectively, and $h(r)$ is a function which should satisfy the two following equations resulting from the equations (21) and (22)

$$
-h(r)-r h^{\prime}(r)=\frac{\rho(r)}{4 \lambda-1} r^{2}
$$

and

$$
-r h^{\prime}(r)-\frac{1}{2} r^{2} h^{\prime \prime}(r)=\frac{\rho(r)}{4 \lambda-1} r^{2} .
$$

One can consider a general situation where the energy density $\rho(r)$ consists of two parts, one constant part and another asymptotically vanishing part, i.e $\rho(r)=\rho_{*}+\frac{\sigma_{*}}{r^{n}}$ where $\rho_{*}$ and $\sigma_{*}$ are positive constants with the dimension of energy density and energy density times (Length) ${ }^{n}$, respectively. Then regarding the equation (24), the solution for $h(r)$ function will be

$$
h(r)=\alpha r^{2}+\frac{\sigma}{r^{m}}
$$

where $\sigma=\frac{\sigma_{*}}{(m-1)(4 \lambda-1)}$ and $\alpha=-\frac{1}{3} \frac{1}{4 \lambda-1} \rho_{*}$ are integration constants. Then, substituting $h(r)$ in (25), one finds two possibilities for $m$, for the consistency of the equation (25), as $m=1$ and $m=-2$. Because $\sigma$ diverges for the case of $m=1$, this case has no physical meaning and can be discarded. For the case of $m=-2$ or equivalently $n=0$, one arrives at $h(r)=\Lambda r^{2}$ where $\Lambda$ is the cosmological constant as $\Lambda=-\frac{1}{3} \frac{1}{4 \lambda-1}\left(\rho_{*}+\sigma_{*}\right) \equiv-\frac{1}{3} \frac{1}{4 \lambda-1} \rho_{0}$. Then, the only possibility is the constant energy density $\rho=\rho_{0}$ representing the vacuum energy or the cosmological constant which we previously obtained its effective equation of state parameter $\omega=-1$ using the consistency of the field equations (20)-(22) of the Rastall theory of gravity. Also, note that here the $\lambda=1 / 4$ parameter leads to a divergent cosmological constant and then is not physically acceptable as we previously mentioned. Finally, the metric will take the following form

$$
\begin{aligned}
d s^{2}= & -\left(1-\frac{2 M}{r}+\frac{Q^{2}}{r^{2}}-\Lambda r^{2}\right) d t^{2} \\
& +\frac{d r^{2}}{1-\frac{2 M}{r}+\frac{Q^{2}}{r^{2}}-\Lambda r^{2}}+r^{2} d \Omega_{2}^{2} .
\end{aligned}
$$

Then, we summarize our results in the following points:

- The metric 27) represents the ReissnerNordström black hole in the cosmological constant background which we obtained in the the context of the Rastall gravity.

- Note that the cosmological constant arises from the consistency of the non-vacuum field equations of the Rastall theory for a spherical symmetric spacetime rather than its ad-hoc introduction to usual Einstein and EinsteinMaxwell field equations. It is also interesting that in the framework of this theory, the Rastall coupling $\lambda$ affects the value of the cosmological constant. Regarding the weak energy condition, i.e $\rho_{0} \geq 0$, for $\lambda<\frac{1}{4}$ we have 
positive cosmological constant representing de Sitter space while for the $\lambda>\frac{1}{4}$ we have a negative cosmological constant denoting anti- de Sitter space.

- Regarding the equations (20)-(22), it is easy to check that for $T_{\mu \nu}^{*}=0$ or equivalently $\rho=p=$ 0 , we arrive at the usual Reissner-Nordström black hole solution

$d s^{2}=-\left(1-\frac{2 M}{r}+\frac{Q^{2}}{r^{2}}\right) d t^{2}+\frac{d r^{2}}{1-\frac{2 M}{r}+\frac{Q^{2}}{r^{2}}}+r^{2} d \Omega_{2}^{2}$.

- Regarding the equations (20)-(22), for the vacuum solution, i.e $T_{\mu \nu}^{*}=E_{\mu \nu}=0$ or equivalently $\rho=p=Q=0$, we arrive at the Schwarzschild solution

$$
d s^{2}=-\left(1-\frac{2 M}{r}\right) d t^{2}+\frac{d r^{2}}{1-\frac{2 M}{r}}+r^{2} d \Omega_{2}^{2} .
$$

- Regarding the equations (20)-(22), for the the case of $T_{\mu \nu}^{*} \neq 0$ and $E_{\mu \nu}=0$ or equivalently $\rho, p \neq 0$ and $Q=0$, we recover the Schwarzschild-(anti)de Sitter solution

$d s^{2}=-\left(1-\frac{2 M}{r}-\Lambda r^{2}\right) d t^{2}+\frac{d r^{2}}{1-\frac{2 M}{r}-\Lambda r^{2}}+r^{2} d \Omega_{2}^{2}$.

Such a solution is obtained as the nontrivial static spherically symmetric vacuum solution of the Rastall theory by solving the equation
$R_{\mu \nu}=\frac{1}{4} R g_{\mu \nu}$ which is resulted by the appropriate setting of the parameters of Rastall theory [19].

\section{CONCLUSION}

We obtained the general spherical symmetric Reissner-Nordström black hole in the cosmological constant background in the the context of the Rastall gravity. In this context, the cosmological constant arises naturally from the consistency of the non-vacuum field equations of the Rastall theory for a spherical symmetric spacetime rather than its adhoc introduction in the usual Einstein and EinsteinMaxwell field equations. It is also interesting that in the framework of this theory, the Rastall coupling $\lambda$ affects the value of the cosmological constant. For $\lambda<\frac{1}{4}$ we have positive cosmological constant representing de Sitter space while for the $\lambda>\frac{1}{4}$ we have a negative cosmological constant denoting anti- de Sitter space. We also discussed about the usual Reissner-Nordström, Schwarzschild and Schwarzschild-(anti)de Sitter black hole solutions as the independent subclasses of the obtained general solution.

\section{ACKNOWLEDGMENTS}

This work has been supported financially by Research Institute for Astronomy and Astrophysics of Maragha (RIAAM) under research project No. 1/4165-84.
[1] S. Nojiri, S. D. Odintsov, Phys. Lett. B 599, 137 (2004).

[2] G. Allemandi, A. Borowiec, M. Francaviglia, S. D. Odintsov, Phys. Rev. D 72, 063505 (2005).

[3] T. Koivisto, Class. Quant. Grav. 23, 4289 (2006).

[4] O. Bertolami, C. G. Boehmer, T. Harko, F. S. N. Lobo, Phys. Rev. D 75, 104016 (2007).

[5] T. Harko, F. S. N. Lobo, Galaxies, 2, 410 (2014).

[6] P. Rastall, Phys. Rev. D 6, 3357 (1972).

[7] G. W. Gibbons, S. W. Hawking, Phys. Rev. D 15, 2738 (1977).

[8] L. Parker, Phys. Rev. D 3, 346 (1971); D 3, 2546 (1971).

[9] L. H. Ford, Phys. Rev. D 35, 2955 (1987).

[10] N. D. Birrell, P. C. W. Davies, Quantum Fields in Curved Space (Cambridge University Press, Cambridge, 1982).

[11] Ph. Brax, C. van de Bruck, A. Davis, arXiv:0706.1024 (2007).

[12] L. L. Smalley, Il Nuovo Cimento B, 80, 1, 42 (1984).

[13] Al-Rawaf, A. S., and Taha, M. O, Phys. Lett. B 366, 69 (1996).

[14] Al-Rawaf, A. S., and Taha, M. O, Gen. Rel. Grav. 28, 935 (1996).
[15] C. E. M. Batista, M. H. Daouda, J. C. Fabris, O. F. Piattella, D. C. Rodrigues, Phys. Rev. D 85, 084008 (2012).

[16] H. Moradpour, Phys. Lett. B 757, 187 (2016).

[17] H. Moradpour, I. G. Salako, AHEP, 2016, 3492796 (2016).

[18] A. F. Santos, S. C. Ulhoa, Mod. Phys. Lett. A 30, 1550039 (2015).

[19] A. M. Oliveira, H. E. S. Velten, J. C. Fabris, L. Casarini, Phys. Rev. D 93, 124020 (2016).

[20] K. A. Bronnikov, J. C. Fabris, O. F. Piattella and E. C. Santos, arXiv:1606.06242.

[21] H. Moradpour, N. Sadeghnezhad, arXiv:1606.00846.

[22] S. Fernando and D. Krug, General Relativity and Gravitation 35, 129 (2003); S. Capozziello, P. A. Gonzalez, E. N. Saridakis and Yerko Vasquez, JHEP 1302, 039 (2013); K.C. K. Chan, J. H. Horne and R. B. Mann Phys. lett. B 447, 23 (1995); E. Babichev, A. Fabbri, JHEP 07,016 (2014); A. Sheykhi, Phys. Rev. D 86, 024013 (2012); D. Garfinkle, G. T. Horowitz, and A. Strominger Phys. Rev. D 43, 3140 (1991). 